\title{
APLICAÇÃO DE TESTES ESTATÍSTICOS NA IMPLANTAÇÃO DOS NOVOS SISTEMAS DE AMOSTRAGEM DO MANUSEIO DE CARVÃO E COQUE DA COMPANHIA SIDERÚRGICA PAULISTA
}

\author{
Sílvio Pereira Diniz Maranha 1 \\ João Paulo Maraia 2 \\ José Roberto Silva de Paiva 3 \\ Plinio Gago 4 \\ Geso Cândido da Silva 5
}

\begin{abstract}
Resumo
Foram implantadas melhorias nos procedimentos e nos equipamentos de amostragem existentes dos diversos sistemas de manuseio de carvão e coque da COSIPA, visando, não só a melhor eficiência técnica dos métodos de retirada dos incrementos para composição das amostras, como também, a melhoria nas condições de segurança operacional da atividade. Através da maior mecanização e automatização das operações de amostragem, foram reduzidos os riscos de acidentes com pessoas. Para avaliar a eficiência técnica das melhorias implantadas nos sistemas de amostragem, foram aplicados Teste de BIAS, conforme N orma ISO 9411, e testes estatísticos comparativos entre pares de amostras, visando a comparação entre os métodos aplicados anteriormente e os atuais.
\end{abstract}

Palavras-chave: Estatística; Segurança; Q ualidade; Amostragem.

\section{STATISTIC METHODS APPLIED TO START UP OF NEW SAMPLING COAL AND COKE HAN DLIN G FACILITIES AT COMPANHIA SIDERÚRGICA PAULISTA}

\begin{abstract}
Improvements in the procedures of sampling and the existing equipment of sampling of the diverse systems of handling of coal and coke of CO SIPA, aiming at, not only, the best efficiency technique of the methods of withdrawal of the increments for composition of the samples, as also, and mainly, improving safety conditions of the activity had been implanted. Due to the great mechanization and automatization of the operations of sampling, the risks of accidents with people had been reduced. To evaluate the efficiency of the improvements implanted in the sampling systems, Test of BIAS BIN DIN G, as ISO 9411 standard, and comparative statistical tests between pairs of samples, have been applied aiming at the comparison between the previously applied methods and the current ones.
\end{abstract}

Key words: Statistics; Safety; Q uality; Sampling.

\section{INTRODUÇÃO}

Em uma grande siderúrgica integrada a coque como a COSIPA, são vários os sistemas de correias transportadoras para o manuseio das matérias primas que, em seus diversos estágios de preparação das matérias-primas para os processos subsequentes, precisam passar por um rígido controle de qualidade. São necessárias retiradas de amostras representativas dos produtos destes processos para que sejam feitas as análises em laboratório e, a partir dos resultados, sejam realizados os necessários ajustes nos processos para adequar sua qualidade. A COSIPA investiu em seus sistemas de amostragem da área de coqueria, implantando melhorias, como amostradores automáticos, visando aprimorar o controle de seus processos de preparação de matérias primas ao mesmo tempo em que reduziu significativamente os riscos de acidentes com seus operadores.

A implantação de melhorias em sistemas de amostragem exigem, além da implantação física dos equipamentos, estudos técnicos, utilizando ferramentas estatísticas, para verificar a boa representatividade das amostras retiradas por estes sistemas. 0 objetivo deste trabalho é mostrar as melhorias implantadas e as técnicas utilizadas, tais como o Teste de BIAS, conforme ISO 9411, e testes de hipóteses de comparação de médias, bem como as técnicas utilizadas para realizar estes estudos.

\footnotetext{
1 M embro da ABM, Analista de Operação da Gerência de Suporte Técnico da Redução da COSIPA

2 M embro da ABM, Analista de Manutenção da Gerência de Suporte Técnico da Redução da COSIPA

3 Membro da ABM , Assistente de Operação da Gerência de Suporte Técnico da Redução da COSIPA

${ }^{4}$ Supervisor da Gerência de Manuseio de Carvões, Coque e PCI da COSIPA

5 Supervisor da Gerência de Manuseio de Carvões, Coque e PCI da COSIPA
} 


\section{DESEN VOLVIMENTO E RESULTADOS}

1 Melhoria no sistema de recebimento de matérias primas (Sistema A):

A COSIPA recebe a maior parte das matérias primas para utilização nas coquerias e no $\mathrm{PCl}$, pelo modal marítimo através de seu porto privativo. 0 sistema de recebimento é composto de 2 linhas de correias transportadoras com capacidade de $1.200 \mathrm{t} / \mathrm{h}$ cada, que possuíam sistemas de amostragem que iniciaram suas operações simultaneamente ao sistema de correias transportadoras em 1986.

Eram necessários melhoramentos nestes sistemas devido a:

- a importância comercial do sistema de amostragem

- o amostrador original tipo corte de fluxo apresentava muitos problemas de manutenção.

- tamanho dos incrementos excessivos de cerca de $80 \mathrm{Kg}$ cada (Foram implantados amostradores do tipo Cross-belt),

0 teste de BIAS, conforme recomendação da N O RMA ISO 9411 - parte 1, foi aplicado na implantação deste sistema. 0 teste é composto de 7 etapas básicas conforme segue:

\subsection{Condições O peracionais}

Através da comparação de pares de amostras coletadas com a CT parada, considerada como referência padrão e pelo amostrador, verificamos se o sistema mecanizado de amostragem implica em algum tipo de vício nos resultados de análise, principalmente granulométricos. A Figura 1 mostra como foi o esquema de coleta dos pares de incrementos, no total de 30 pares, seguindo as orientações da norma ISO 9411.

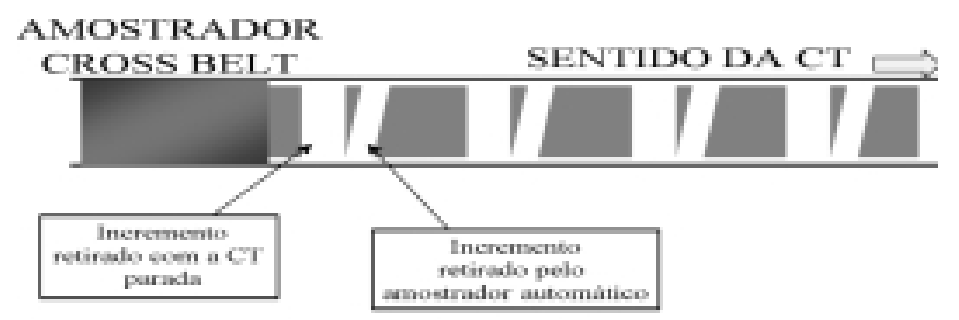

Figura 1. Esquema de coleta dos pares de incrementos.

\subsection{Estatística Básica}

Tabela 1. Estatística básica para os principais parâmetros comparados.

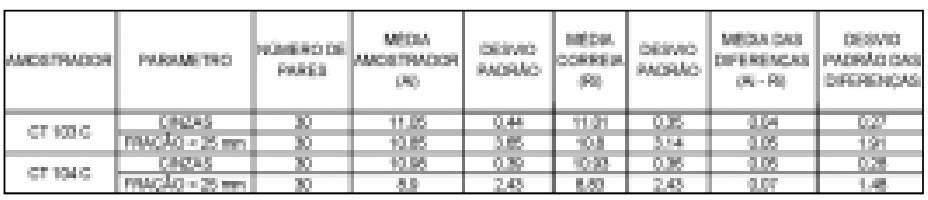

\subsection{Análise Gráfica dos Resultados}

As Figuras 3 e 4 mostram exemplos dos gráficos de dispersão, feitos para todos os parâmetros analisados, para ajudar na identificação de pontos muito discrepantes da massa de dados (O UTLIERS), tendenciosidade, falta de controle estatístico e vícios.

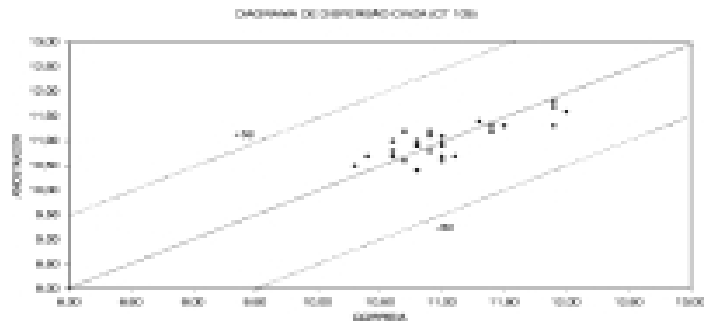

Figura 3. Diagrama de dispersão do parâmetro cinza (amostrador 103C).

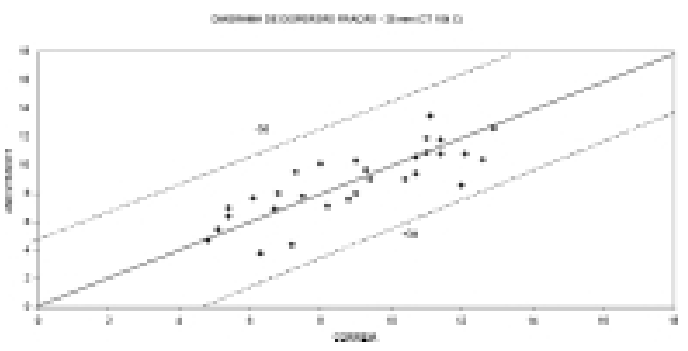

Figura 4. Diagramas de dispersão do parâmetro $-25 \mathrm{~mm}$ (amostrador 104C).

\subsection{Verificação de "OUTLIERS": Teste COCHRAN}

Este procedimento é baseado no critério de máxima variância de COCHRAN, cujo critério é baseado na seguinte equação:

\begin{tabular}{|c|c|c|}
\hline $\mathbf{C}=\mathrm{d}^{2}$ máx & $\begin{array}{l}\text {, onde: di } \\
\qquad \text { dmax }\end{array}$ & $\begin{array}{l}\text { é o valor da diferença } \\
\text { é o maior valor }\end{array}$ \\
\hline $\bar{n}$ & & absoluto do conjunto \\
\hline $\begin{array}{l}\sum \mathrm{d} 2 \mathrm{i} \\
\mathrm{i}=1\end{array}$ & $\mathbf{n}$ & $\begin{array}{l}\text { de dados } \\
\text { é o número de pares } \\
\text { do conjunto de dados }\end{array}$ \\
\hline
\end{tabular}

A Tabela 2 a seguir mostra os resultados do teste Cochran para os parâmetros analisados.

Tabela 2. Resultados do teste de Cochran.

\begin{tabular}{|c|c|c|c|c|c|}
\hline 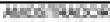 & Phow IND & 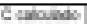 & $\cos \theta$ & 05 & Ecowsesta \\
\hline $\operatorname{cting}$ & cins & $0 . \bar{x}$ & $0 x$ & $\cos 2+\cos$ & W000 \\
\hline & 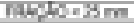 & 98 & $9 x$ & $\cos =00^{\circ}$ & 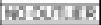 \\
\hline crise & $\cos 2$ & क्तi & $0 x$ & $\cos =0$ & Feroulxal \\
\hline & $20083 \mathrm{~mm}$ & Skit & 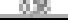 & $\cos 2 \cdot \cos ^{2}$ & Herounders \\
\hline
\end{tabular}

Pelo teste de Cochran, confirmando as análises gráficas anteriores, não ocorreram "outliers" entre os pares coletados.

\subsection{Cálculo do Número de Pares Necessários}

O cálculo do número de pares necessários é dados pela equação:

$$
g=\frac{M T B}{S d} \quad \begin{aligned}
\text {, onde: MTB é valor máximo de Bias } \\
\text { Tolerado pré tabelado }
\end{aligned}
$$


A tabela abaixo mostra quantos pares seriam necessários e quantos foram retirados, mostrando que o número de pares coletados foram suficientes para o nível máximo de BIAS tolerados.

Tabela 3. Resultados do cálculo de g.

\begin{tabular}{|c|c|c|c|c|c|c|}
\hline mosenvest & nuwerse & $\mathrm{sn}$ & so & $\bullet$ & $x^{200}$ & ronisus \\
\hline cr $\approx c$ & 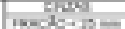 & the & s. & 30 & 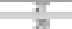 & $x$ \\
\hline of $=4 \mathrm{c}$ & करेण & i: & 28 & 18 & 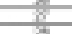 & 3 \\
\hline
\end{tabular}

\subsection{Análise de Significância: Teste t}

0 teste $t$ verifica a existência de diferença entre as médias das amostras, caracterizando ou não a existência de BIAS. A tabela a seguir mostra a estatística :

Tabela 4. Resultados do teste t.

\begin{tabular}{|c|c|c|c|c|c|}
\hline 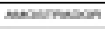 & 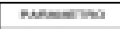 & indestis & $=$ & mat a & cincoinas \\
\hline \multirow{2}{*}{$e n$} & eness & 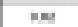 & $a n$ & teastive & west \\
\hline & incostoram & sas & $2 n$ & 1estitin & $m \rightarrow \ln ^{2}$ \\
\hline \multirow{2}{*}{ Cr wate } & cantan & in & $2 m$ & $100+10$ & 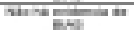 \\
\hline & 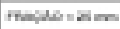 & ka & 23 & thentive & $x^{2}$ \\
\hline
\end{tabular}

\subsection{Avaliação Final de BIAS} equação:

A variável Razão de BIAS (BR) é dada pela $B R=\frac{-d}{\text { MTB } \times 0,545}$, onde: ${ }^{-} d$ valor da diferença média

A Tabela 5 mostra os valores calculados de BR para os três parâmetros analisados.

Tabela 5. Análise do Fator de Bias para os parâmetros da mistura de carvões.

\begin{tabular}{|c|c|c|c|c|c|c|}
\hline & 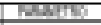 & 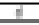 & in & e & $\operatorname{lnth}$ & onanes \\
\hline \multirow{2}{*}{$5 x$} & $a=$ & $\Rightarrow$ & $m$ & 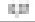 & $t<\mathbf{m}<1$ & 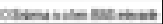 \\
\hline & redorzan & $\mathrm{E}$ & 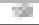 & $6 x$ & महले & 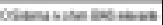 \\
\hline \multirow{2}{*}{500} & $\mathrm{Cos}$ & 恶 & Es & $4 x$ & 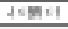 & 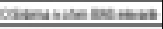 \\
\hline & pecour $7=$ & $\Rightarrow$ & $=$ & te & $\{=0.1$ & 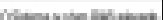 \\
\hline
\end{tabular}

\section{MELHORIA NO SISTEMA DE ENVIO DE MISTURA DE CARVÃO (SISTEMA C)}

As melhorias neste sistema eram necessárias devido a:

- As condições de segurança do operador para manuseio das amostras eram inadequadas.

- 0 amostrador apresentou vício em alguns parâmetros a partir de estudo prévio realizado, após as alterações no sistema.

- 0 custo de um novo amostrador era de US\$ 50.000 .
Foi testado aumento da seção de coleta da colher pela adição de canecas auxiliares laterais.
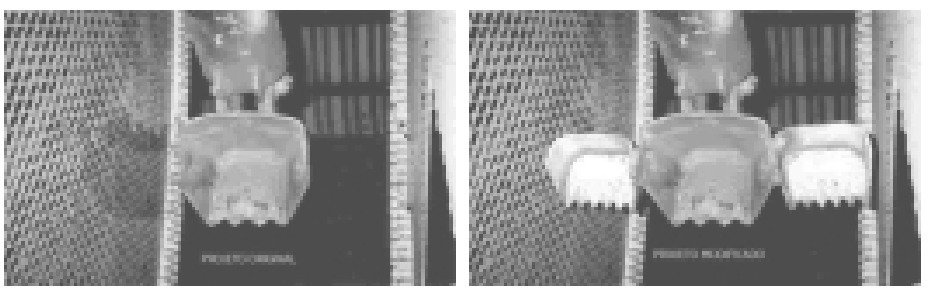

Figura 5. Caneca do amostrador antes e após a melhoria.

A pós as modificações foi realizado também o Teste de BIAS conforme ISO 9411.

\subsection{Condições 0 peracionais}

O teste foi realizado no período de 19/05/98 a 20/05/98 quando foram retirados 30 pares de amostras coletados a cada 30 minutos. As amostragens de referência foram realizadas com a correia parada sendo coletadas a cerca de $10 \mathrm{~cm}$ do corte realizado pelo amostrador com um gabarito de $100 \mathrm{~mm}$ de abertura. As amostras foram integralmente processadas nas peneiras de $3,36 \mathrm{~mm}, 0,50 \mathrm{~mm}, 0,105 \mathrm{~mm} \mathrm{e}<0,105 \mathrm{~mm}$, que foram reduzidas para determinação de Cinza e Matéria Volátil.

\subsection{Estatística Básica}

A Tabela 6 mostra os resultados estatísticos dos resultados das amostras de referência (Ri) e das amostras coletadas pelo amostrador (Ai).

Tabela 6. Estatística básica dos resultados analisados.

\begin{tabular}{|c|c|c|c|c|c|c|}
\hline PARNAETRO & $\begin{array}{c}\text { NEDAA } \\
\text { AMOSTRADOR } \\
\text { (AN) }\end{array}$ & $\begin{array}{l}\text { DESVIO } \\
\text { PADFOSO }\end{array}$ & $\begin{array}{c}\text { NFOA } \\
\text { COFREA } \\
\text { (vi) }\end{array}$ & $\begin{array}{l}\text { DESMO } \\
\text { PADR:SO }\end{array}$ & $\begin{array}{l}\text { NEDDA DAS } \\
\text { DFERENCAS } \\
(\boldsymbol{N}-\mathbf{R})\end{array}$ & $\begin{array}{c}\text { OESVOO } \\
\text { PADRLIO } \\
\text { QLS } \\
\text { DIFEFENCAS }\end{array}$ \\
\hline CINDS & 792 & $0.2 \%$ & 792 & 0.27 & 0.01 & 0292 \\
\hline NATERAYOLATLL & 29.34 & 0.691 & 24,49 & 0.92 & $.0,06$ & 0342 \\
\hline FRAC $50<335 \mathrm{~mm}$ & 7785 & 2,587 & $77 n$ & 189 & 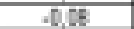 & 2.112 \\
\hline FRWC200060 m & $30 \%$ & $4,1 \pi$ & $3.4)$ & 4,45 & 0.12 & 2597 \\
\hline FAC $10 \mathrm{~s} .125 \mathrm{~mm}$ & $8 \mathrm{Bg}$ & 3.034 & 9.43 & 2.01 & 0,20 & 244 \\
\hline
\end{tabular}

\subsection{Análise Gráfica dos Resultados}

Plotados mas não apresentados nesta versão.

\subsection{Verificação de "OUTLIERS": Teste COCHRAN}

Tabela 7. Tabela comparativa de COCHRAN para os parâmetros analisados.

\begin{tabular}{|c|c|c|c|c|}
\hline PSQDWETRO & Cevoulach & Caticocys: & Ot5 & ConcLUERO \\
\hline CNDS & 0.16 & 0.365 & Caic \& Cut & NOOMUER \\
\hline WATERIA WOLATL & 0005 & 0,363 & $\operatorname{Cos}<<C a x$ & WOOUTUER \\
\hline PRSSENTE 3,S5Mm & 0.105 & 0,363 & $\operatorname{Coac}<\mathrm{Cax}$ & WOOUTUER \\
\hline PRSSUNTE O.50mm & 0.10 & 0,263 & $\operatorname{Cos} 1<\cos t$ & NOONTUE \\
\hline PASSANTEL.7L5m & 0005 & 0,365 & Care <Cwh & WOOJTLER \\
\hline
\end{tabular}

Pela comparação entre valores calculados e tabelados da variável estatística $C$ não existem OUTLIERS para as determinações realizadas 


\subsection{Cálculo do N úmero de Pares Necessários}

Tabela 8. Número de pares para cada parâmetro analisado necessário para continuar o teste estatístico.

\begin{tabular}{|c|c|c|c|c|}
\hline PMFWVETAO & MTE & इ0 & 9 & 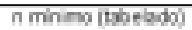 \\
\hline CFULS & 020 & 0.292 & 0.002 & 28 \\
\hline METLFA WOLATL & $a>3$ & 032 & 0,731 & $2 x$ \\
\hline FRECIO $<835 \mathrm{~mm}$ & 180 & 2.112 & 0.710 & 28 \\
\hline TAAC,ADVOSO $\mathrm{mm}$ & 150 & 2,391 & 0,50 & 41 \\
\hline 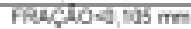 & 150 & 2.491 & aहाइ & 56 \\
\hline
\end{tabular}

\subsection{Análise de Significância : Teste t}

Tabela 9. Resultados das estatísticas t de comparação de médias entre as amostras de referencia e as coletadas no amostrador.

\begin{tabular}{|c|c|c|c|c|}
\hline PMFAVETRO & tcalousds & t tabecaso $35 \mathrm{~h}$ & ANRUSE & CONCLUSIS \\
\hline CINZAS & 0,000 & 1,460 & teric \& t tat & Nab hal evidena a de bue \\
\hline MATERA VDLATIL & 1.019 & 1,060 & teric \& t tat & Naba hat evidendia de DuC \\
\hline FARCAO $<3.36 \mathrm{~mm}$ & 0,190 & 1,656 & teacestat & 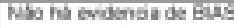 \\
\hline
\end{tabular}

A tabela mostra que não existe mais evidência de BIAS no amostrador após as modificações.

\subsection{Avaliação Final de BIAS}

Tabela 10. Análise do Fator de Bias para os parâmetros da mistura de carvões.

\begin{tabular}{|c|c|c|c|c|c|}
\hline 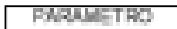 & 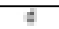 & NTIS & EF & Dowbse & Conctustor \\
\hline कासटबड & $0, \mathrm{C}$ & 0.200 & 0,4 & $-1<B<<1$ & 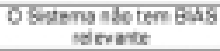 \\
\hline NA,TEAA VOUTL & $-6,09$ & 0.250 & $-0,19$ & $-1<B<1$ & 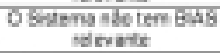 \\
\hline $\begin{array}{c}\text { FFOACIOS } \\
\mathrm{mm}\end{array}$ & 6,09 & 1500 & $-0,0$ t & $-1<B<<1$ & 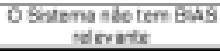 \\
\hline
\end{tabular}

\section{MELHORIA NO SISTEMA DE ENVIO DE COQUE PARA OS ALTOS FORNOS}

Foram implantados dois amostradores tipo Cross-Belt nas CTs T2 (Manuseio de Coque I) e 251 (Manuseio de Coque II).

Foi realizado teste comparativo de resultados entre as amostras coletadas na rampa 2 (procedimento manual padrão) e no amostrador da CT 251 (coletando incrementos a cada 20 minutos), A Tabela 11 resume os resultados obtidos neste estudo.

Tabela 11. Resultados comparativos dos pares de amostras de coque.

\begin{tabular}{|c|c|c|c|c|c|c|}
\hline \multicolumn{7}{|c|}{ 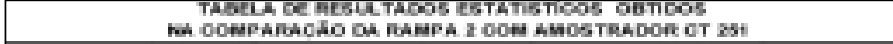 } \\
\hline & & & & & LEENT & \\
\hline PRAOAELINOO & to chi & 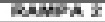 & AW Cr 251 & CALO & TRGCLOO & CCONECLSTE \\
\hline cowoses) & x & 970 & 304 & $1 \pi 0$ & 100 & DERENENTER \\
\hline MV A & 30 & Q.aा & 9.03 & ax & 1.00 & GLWA \\
\hline 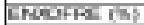 & 36 & बar & वर्क & 1.19 & 100 & DELA \\
\hline MIOACe Cots & wo & 23 & 30 & $-5 \times 0$ & 1.09 & DOE ENENTES \\
\hline TM imró & 36 & 64.7 & 91.4 & 2021 & 1.60 & DEFERENTES \\
\hline 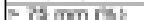 & 30 & 20.1 & 50.1 & $2 \pi, 7$ & 100 & DEFLAINILE \\
\hline रू $\mathrm{cm}$ (ब) & IE & 12 & 73 & -13 两 & 160 & DE-ERLNTE: \\
\hline CMा & 20 & 29,0 & 242 & $x \geq 2$ & $1 \pi 1$ & DER NENTER \\
\hline Can & $\pi$ & $64 \pi$ & ab, & an & $1 \pi$ & moLasa \\
\hline 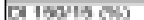 & 36 & 824 & 53.6 & $-0,00$ & 1.00 & DERERENTES \\
\hline
\end{tabular}

\section{CONCLUSÕES}

1 As melhorias implantadas nos sistemas de amostragem da CO SIPA visaram, além de um melhor desempenho técnico da amostragem, a redução de riscos para os operadores

2 A implantação dos amostradores foram acompanhadas de critérios técnicos, que foram testados na escala industrial seguindo procedimentos normalizados.

30 s resultados obtidos mostraram que os sistema implantados apresentaram bom desempenho técnico, atendendo às expectativas operacionais e técnicas.

Recebido em: 03/02/2006

Aceito em: 22/09/2006

Proveniente de: SEMINÁRIO DE REDUÇÃO DE MINÉRIO DE FERRO E MATÉRIAS-PRIMAS, 36. e SIMPÓSIO BRASILEIRO DE MINÉRIO DE FERRO, 6., O uro Preto, MG. São Paulo : ABM, 2005. 